\title{
Relación entre habilidades para la vida y riesgos vitales en adolescentes escolarizados de la ciudad de Armenia
}

\section{Relationship between life skills and vital risks in adolescents who attend school in the city of Armenia}

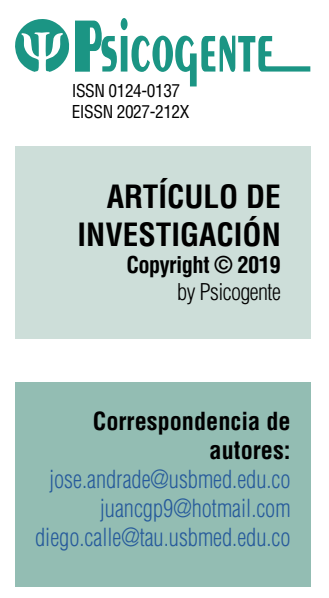

Recibido: $22-01-18$ Aceptado: $25-06-18$ Publicado: 01-07-19

José Alonso Andrade Salazar iD - Juan Gonzales Portillo iD - Diego Alejandro Calle
Sandoval iD
Universidad de San Buenaventura Medellín extensión Armenia, Colombia

Resumen

Objetivo: Analizar la relación entre habilidades para la vida y riesgos vitales en adolescentes escolarizados.

Método: Esta es una investigación cuantitativa descriptiva-correlacional, en la que se aplicó la escala de habilidades para la vida de Díaz, Rosero, Melo y Aponte (2013), y se diseñó un cuestionario de identificación de riesgos vitales y una ficha de caracterización. La muestra fueron 107 estudiantes (adolescentes entre 12 y 17 años) de $9^{\circ}, 10^{\circ}$ y $11^{\circ}$ de bachillerato de una institución educativa pública de la ciudad de Armenia, y fueron seleccionados a través de un muestreo no probabilístico.

Resultados: En las habilidades para la vida prevalecen: empatía (65,4 \%), toma de decisiones $(57,9 \%)$, solución de problemas y conflictos (62,6\%). Los riesgos psicosociales identificados son: relacional (81,3\%), intergrupal (95,3\%), personal (97,2\%), psicológico (96,3\%), y comunitarios (96,3\%). Existe correlación entre manejo de sentimientos y emociones, tensiones y estrés con regulación emocional y reconocimiento de fuentes de estrés $(p=0,566)$; manejo de tensiones y estrés, y comunicación efectiva y asertiva con humildad y estrategias específicas $(p=0,5)$ como respirar profundamente y tener pensamientos saludables $(p=0,605)$.

Conclusiones: Una relación sana con los padres mejora sus habilidades para la vida, pero disminuye el reconocimiento de riesgos en el hogar y el ambiente; interacciones conflictivas con los padres afectan las habilidades para la vida; manejar sentimientos y emociones se relaciona con la regulación y expresión emocional, así, quien aprehende afectivamente a explorar su vida emocional tiene mejores habilidades para identificarlas, implementarlas y expresarlas al momento de tomar decisiones.

Palabras clave: adolescencia, habilidades para la vida, conductas de riesgo, riesgo social, riesgo ambiental, riesgo familiar.

Abstract

Objective: This paper aims to analyze the relationship between life skills and vital risks in adolescents who attend school.

Method: This is a quantitative correlational descriptive-research; in this research a scale based on life skills by Diaz, Rosero, Melo and Aponte (2013) was applied, and also a vital risk identification questionnaire and a sheet of characterization were designed. 107 from ninth, tenth and eleventh grade students (adolescents aged 12-17) of a public high school from Armenia (Col) through a non-probabilistic were selected and sampled.

Results: As result, life skills such as: empathy (65,4\%), decision-making (57,9\%), problem and conflict solving (62,6\%). Identified psychosocial risks are, as follows: relational (81,3\%), intergroup (95,3\%), staff $(97,2 \%)$, psychological $(96,3 \%)$, community $(96,3 \%)$. There is a correlation between feelings and emotions, tensions and stress with emotional regulation and recognition of sources of stress management $(p=0,566)$; tension and stress management, and effective and assertive communication with humility $(p=0,5)$ and specific strategies such as breathing deeply and thinking healthy thoughts $(p=0,605)$.

Conclusions: A healthy relationship with parents improves life skills, but the recognition of risks at home and the environment decreases. Conflicting interactions with parents affect life skills. To cope with feelings

Cómo citar este artículo (APA):

Andrade Salazar, J.A., Gonzales Portillo, J. \& Calle Sandoval, D.A. (2019). Relación entre habilidades para la vida y riesgos vitales en adolescentes escolarizados de la ciudad de Armenia. Psicogente 22(42), 1-23. https://doi.org/10.17081/psico.22.42.3490 

decisions

Key words: adolescence, life skills, risk behaviours, social risk, environmental risk, family risk.

\section{INTRODUCCIÓN}

Los adolescentes se encuentran expuestos a notables y distintos riesgos psicosociales que dificultan no solo sus procesos de ajuste psicosocial sino también la capacidad de responder adecuadamente ante el estrés (Andrade \& Gonzales, 2012); en contraste, los factores protectores se componen también de habilidades para la vida, las cuales se articulan unas con otras, orientan el desarrollo de nuevas habilidades protectoras, programas de prevención y estrategias de contención de comportamientos auto-destructivos. Entre los riesgos frecuentes a los que están expuestos los adolescentes se encuentran: medioambiente estresor, familias desprotectoras, negligentes, disfuncionales, agresivas o inter-excluyentes (Herrera-Santí, \& Avilés-Betancourt, 2000), ambiente escolar desprotector, tenso o peligroso (Alfonso, Calcines, Monteagudo de la Guardia, \& Nieves-Achon, 2015; Omar, Almeida da Silva, Paris, Aguilar de Souza \& del Pino Peña, 2010); ambiente comunitario de elevado riesgo para el desarrollo integral, susceptibilidad a influencias externas (Quiceno \& Vinaccia, 2014). Las vulnerabilidades ante comportamientos riesgosos ponen en juego las llamadas «habilidades protectoras» (Castro \& Casullo, 2000), estas destrezas implican todo el repertorio conductual y cognoscitivo de la persona, además que se dan en el marco de la psicohistoria.

Los modelos psicológicos actuales, proponen por medio de un cambio de paradigma ontológico la percepción del sujeto desde modelos biopsicosociales, de modo que la psicopatología y sus factores de riesgo asociados se entienden en el marco de una visión mucho más amplia. Tal es el caso de la concepción neuropsicológica funcionalista del pensamiento ejecutivo y en especial de la toma de decisiones, la cual se vincula estrechamente con la ideación e intento suicida (Calle \& Gonzales, 2016), así como en la génesis del desarrollo del control voluntario de la conducta y el pensamiento (Calle, 2016). Dentro del desarrollo de una investigación realizada, Góngora y Casullo (2009) proponen como factores protectores de la salud mental tres variables: inteligencia emocional, valores y autoestima. Según estos autores: "en Psicología Clínica los aspectos positivos son abordados en dos formas principales: por un lado, como factores a fortalecer en momentos de 
salud [...] y por otro, el trabajo de fortalecimiento de los aspectos positivos también resulta terapéutico" (p.186). Las habilidades protectoras en los sujetos tienen como objeto primordial la promoción de la salud antes que la prevención, por tanto, dentro de este modelo de habilidades o factores protectores conocidos también como salutogénicos, implican la condición de conocimiento y desarrollo de la integralidad de la persona en relación con sus limitaciones y el conocimiento de posibles factores de riesgo. Este modelo también ha servido de referencia para posteriores investigaciones y han afianzado la evidencia clínica (Góngora \& Casullo, 2009; Quiceno \& Vinaccia, 2013).

En Colombia se ha investigado sobre las habilidades protectoras en relación con factores de riesgo asociados al suicidio; es así como Quiceno y Vinaccia (2013) en una investigación llevada a cabo en una muestra de adolescentes de la ciudad de Bogotá, encontraron que: "los adolescentes que tienen metas claras respecto a su futuro, que se sienten felices y valoran y le dan significado a la vida perciben un mejor bienestar psicológico y relación con sus padres" (p. 263). Las investigaciones han permitido afirmar que lo planteado por Góngora y Casullo (2009) es significativo: el correcto conocimiento y uso de la escala de valores, inteligencia emocional y autoestima, se orientan a ayudar la resiliencia como primer y gran objetivo de los modelos de prevención en salud. Cabe mencionar que el riesgo de mayor peligro vital en los adolescentes es el suicidio (Herrera-Santí, \& Avilés-Betancourt, 2000), y comienza por la ideación suicida como uno de los factores precipitantes, pues se denota como todo pensamiento elaborado que puede suscitar la autoeliminación del sujeto (Goldney, Winefield, Tiggemann, Winefield \& Smith, 1989; Gonzales, Gil, Hernández \& Henao, 2016). Asimismo, la ideación suicida sin planificación de la acción se denomina primaria, y en ella la persona expresa su deseo de morir, pero aún no tiene claridad de cómo hacerlo (Andrade, 2012). Ergo, cuando existe un método inespecífico es secundaria; con método específico no planificado, la ideación suicida es terciaria, y la más peligrosa es la terciaria o plan suicida, porque la persona expresa y está convencida de quitarse la vida, además, ya ha pensado el método, lugar y los pasos para llevarlo a cabo (Goldney et al., 1989; Farberow, 1980; Andrade, 2012). En ocasiones suceden suicidios por imitación cuando un personaje público, un familiar u otra persona desata el interés en personas que han tenido intentos previos de suicidio o que atraviesan por crisis personales y problemas familiares crónicos (Casullo, Bonaldi \& Fernández, 2000; Bahamón et al., 2018). Otro de los riesgos es el parasuicidio o conducta definida como todo intento de suicidio realizado con el fin de llamar la atención, sobre un aspecto de las 
relaciones con otros con quienes a menudo se entra en conflicto o dilema permanente (Maris, 1992; Ferguson, Beautrais \& Horwood, 2003).

En el ámbito social el parasuicidio es poco usado como término, sin embargo intento de suicidio y parasuicidio suelen ser usados indistintamente (Mardomingo \& Catolin, 1992; Welch, 2001). Cabe anotar que no hay cifras fiables sobre las dimensiones del parasuicidio y suele ser asociado a problemas depresivos que se cronifican y elevan los factores de riesgo en las personas (Isometsa, 2001; Lonnquist, 2002), motivo por el cual el riesgo de muerte por parasuicidio suele ser 40 veces mayor (Henriksson, et al., 1993); Además, suele tener una baja letalidad y elevada impulsividad. Es común entre adolescentes, mujeres jóvenes, y personas con rasgos de personalidad depresiva, manipuladora o chantajista a nivel emocional (Lonnquist, 2002). Por tanto, el desarrollo de habilidades para la vida constituye una herramienta para el crecimiento personal y social, ya que incrementa en los sujetos la capacidad para vivir mejor y transformar las condiciones de desigualdad socio-cultural (Mantilla \& Chahin, 2006). La enseñanza de habilidades para la vida previene problemas psicosociales (Espada, Méndez, Griffin \& Botvin, 2003; Mantilla, 2001), permite también ampliar el conocimiento y la información disponible sobre la forma como se pueden prevenir conductas de riesgo, así como también el trabajo inter-pedagógico entre familia-comunidad-institución (Mantilla, 2001). De suyo, los programas de habilidades para la vida y de robustez de factores protectores de la vida (Bravo, 2005), deben estar dirigidos a prevenir múltiples problemáticas sociales y de salud, al tiempo que fortalece las herramientas y estrategias ya implementadas por personas y comunidades (Bravo \& Garzón, 2010).

La Organización Panamericana de la Salud (OPS) a través de su enfoque de habilidades para la vida para un desarrollo saludable de niños y adolescentes, señala que existen tres bloques de habilidades básicas y necesarias que no se emplean forma individual, ya que se complementan y refuerzan mutuamente (Mangrulkar, Whitman, \& Posner, 2001). Estas habilidades son: habilidades sociales (de comunicación, negociación/rechazo, de aserción, interpersonales, de cooperación y de empatía y toma de perspectivas); habilidades cognoscitivas (toma de decisiones/solución de problemas, comprensión de las consecuencias de las acciones, determinación de soluciones alternas para los problemas, de pensamiento crítico, análisis de la influencia de sus pares y de los medios de comunicación, análisis de las propias percepciones de las normas y creencias sociales y autoevaluación y clarificación de valores; y por último, habilidades para controlar las emociones, control del estrés y control 
de sentimientos para aumentar el locus de control interno. Es preciso anotar que estas habilidades deben tener un contenido informativo con base en tareas personales, sociales y de salud de la adolescencia a través de metodologías interactivas de enseñanza (Mangrulkar, et al, 2001). Las habilidades para la vida (en adelante HV) sirven de factor protector no solo de la ideación suicida, sino también, de otras conductas anómalas o destructivas en las personas, y se constituyen en pilares educativos desde edades tempranas.

\section{MÉTODO}

\subsection{Diseño.}

Esta es una investigación cuantitativa descriptiva-correlacional, cuyo objetivo es analizar la relación entre habilidades para la vida y riesgos vitales en adolescentes escolarizados.

\subsection{Participantes}

La muestra fue de 107 estudiantes -hombres y mujeres- que cursan $9^{\circ}, 10^{\circ}$ y $11^{\circ}$ de bachillerato de una institución educativa (IE) pública del departamento del Quindío. Los participantes fueron seleccionados a través de un Muestreo no probabilístico. Asimismo, los criterios de inclusión fueron: el estar matriculado en la IE, cursar $9^{\circ}, 10^{\circ}$ o $11^{\circ}$ de bachillerato, encontrarse en la etapa de adolescencia (12-18 años). No fueron criterios de exclusión la etnia, el estrato o la religión.

\subsection{Instrumentos}

\subsubsection{Habilidades para la vida (Díaz, Rosero, Melo \& Aponte, 2013)}

Este instrumento presenta una elevada consistencia interna $(\alpha=0,7129)$ lo cual le otorga una buena confiabilidad, además de una validez en tanto correlación variable con las dimensiones: Conocimiento de sí mismo $(\alpha=0,669)$, Empatía $(\alpha=0,755)$; Comunicación efectiva y asertiva $(\alpha=0,615)$; Relaciones interpersonales $(\alpha=0,666)$; Toma de decisiones ( $\alpha=0,662$; Solución de problemas y conflictos $(\alpha=0,583)$; Pensamiento creativo $(\alpha=0,843)$; Pensamiento crítico $(\alpha=0,791)$; Manejo de sentimientos y emociones $(\alpha=0,689)$; Manejo de tensiones y estrés $(\alpha=0,492)$.

La escala de Habilidades para la vida es un instrumento de auto aplicación tipo Likert en el que 80 reactivos dan cuenta de diez dimensiones claves en la adquisición de habilidades para la vida: conocimiento de sí mismo, 
empatía, comunicación efectiva y asertiva, relaciones interpersonales, toma de decisiones, solución de problemas y conflictos, pensamiento creativo, pensamiento crítico, manejo de sentimientos y emociones y manejo de tensiones y estrés. Todas estas a su vez poseen subdimensiones y reactivos con las opciones de respuesta: Siempre (S), Casi Siempre (CS), Algunas Veces $(\mathrm{AV})$, Casi Nunca (CN) y Nunca (N). Cada uno de estas opciones tiene los valores internamente de 5 a 1 ; en el caso de los reactivos con direccionalidad positiva, es decir, 5 equivale a Siempre y 1 a Nunca. Por el contrario, en los ítems con direccionalidad negativa la direccionalidad es la opuesta. Por su parte el cuestionario de factores de conducta de riesgo aplicado consta de 28 ítems para autoaplicación en formato Likert con opción de respuesta nunca, algunas veces, frecuentemente y siempre, que se agrupan en siete dominios o factores de riesgo: riesgo ambiental, riesgo relacional, riesgo psicológico, riesgos comunitarios, riesgos personales, riesgos intergrupales y riesgos familiares. El proceso de validación del cuestionario se realizó con base en el criterio de evaluación de tres jueces expertos, y los criterios de relevancia, suficiencia, claridad y coherencia.

\subsubsection{Ficha de caracterización psicosocial}

Fue sometida a un proceso de validación de constructo con base en la revisión bibliográfica previa, y el criterio de evaluación de tres pares expertos; posterior a ello se realizó el pilotaje de la ficha y se ajustaron los criterios.

\subsubsection{Cuestionario de identificación de factores de conductas de riesgo}

Este cuestionario fue elaborado para la investigación tras una extensa revisión teórica, a través de la validez de constructo, enfocada en acoplar los constructos a grados medibles de significados en torno a la temática tratada. Tanto la ficha de caracterización como este cuestionario fueron sometidos a un pilotaje previo con población adolescente escolarizada.

\subsection{Procedimiento}

La investigación se desarrolló a través de cuatro procedimientos a saber: a) aprobación de la propuesta de investigación y aval del comité de Bioética; b) aplicación de instrumentos y rastreo bibliográfico; c) análisis de datos estadísticos a través del software SPSS 18, en el que se empleó la estadística paramétrica y la prueba de correlación de Pearson, d) redacción informe resultados del estudio descriptivo-correlativo. 


\subsection{Análisis de datos}

El tipo de estadística fue aplicada, ya que con ella se buscó deducir los resultados sobre el universo de estudiantes, tomando como muestra representativa $9^{\circ}, 10^{\circ}$ y $11^{\circ}$ de bachillerato. Para medir la relación entre variables se implementó el coeficiente de correlación de Pearson (inversa: valor negativo; directamente proporcional: valor positivo), la cual se establece de acuerdo a los siguientes intervalos: 1,0 a 0,5/-1,0 a -0,5 correlación fuerte; 0,3 a 0,5/-0,5 a -0,3 correlación moderada; 0,1 a 0,3/-0,3 A -0,1 correlación débil; 0,1/-0,1 ninguna o muy débil correlación.

\subsection{Declaración de aspectos éticos}

Partiendo de las consideraciones éticas señaladas en la Declaración de Helsinki esta investigación tuvo un bajo riesgo, y para llevarla a cabo se solicitó el aval del consentimiento informado al comité de Bioética de la Universidad de San Buenaventura, Medellín, el cual fue firmado por los participantes del estudio previo a la aplicación de los instrumentos. Se indicó a las personas que los resultados tienen un uso investigativo-académico, además de la confidencialidad de la información recopilada, el respeto a su dignidad y la protección de sus derechos y bienestar.

\section{RESULTADOS}

De acuerdo a la ficha de caracterización (tabla 1), los adolescentes tienen edades que van desde 14 (14\%), 15 (29\%), 16 (31,8\%), 17 (17,8\%) y 18 $(7,5 \%)$ años; en $9^{\circ}(25,2 \%), 10^{\circ}(41,1 \%)$, y $11^{\circ}$ grado $(33,6 \%)$; prevalece la religión católica $(56,1 \%)$ y cristiana $(36,4 \%)$, la Unión libre de los padres (41,1\%), los estratos 1 (35,5\%) y $2(47,7 \%)$; la etnia mestiza (98,1\%), el 12,1 $\%$ de trabajo infantil, el contar con una red de apoyo $(63,6 \%)$ y contar con una buena relación con los padres (70,1\%).

Tabla 1.

Datos Ficha de caracterización

\begin{tabular}{cccc}
\hline ÍTEM & & $\mathbf{N}^{\circ}$ & $\%$ \\
\hline & 14 & 15 & 14,0 \\
\multirow{2}{*}{ Edad } & 15 & 31 & 29,0 \\
& 16 & 34 & 31,8 \\
& 17 & 19 & 17,8 \\
& 18 & 8 & 7,5 \\
Grado de escolaridad estudiantes & $9^{\circ}$ & 27 & 25,2 \\
& $10^{\circ}$ & 44 & 41,1 \\
& $11^{\circ}$ & 36 & 33,6 \\
\hline
\end{tabular}




\begin{tabular}{|c|c|c|c|}
\hline ÍTEM & & $\mathbf{N}^{\circ}$ & $\%$ \\
\hline \multirow{4}{*}{ Orientación religiosa } & Católico & 60 & 56,1 \\
\hline & Evangélica & 1 & 0,9 \\
\hline & Cristiana & 39 & 36,4 \\
\hline & Otros & 7 & 6,5 \\
\hline \multirow{3}{*}{ Estado civil de los padres } & Viven juntos & 44 & 41,1 \\
\hline & $\begin{array}{l}\text { No convive } \\
\text { con ellos }\end{array}$ & 2 & 1,9 \\
\hline & Separados & 61 & 57,0 \\
\hline \multirow{5}{*}{ Estrato socioeconómico } & Estrato 1 & 38 & 35,5 \\
\hline & Estrato 2 & 51 & 47,7 \\
\hline & Estrato 3 & 17 & 15,9 \\
\hline & Estrato 4 & 1 & 0,9 \\
\hline & Estrato 5 & 0 & 0,0 \\
\hline \multirow{5}{*}{ Grupo étnico } & Indígena & 1 & 0,9 \\
\hline & Mestizo & 105 & 98,1 \\
\hline & $\begin{array}{l}\text { Afrodescen- } \\
\text { diente }\end{array}$ & 1 & 0,9 \\
\hline & Caucásico & 0 & 0,0 \\
\hline & Otros & 0 & 0,0 \\
\hline \multirow{2}{*}{ Trabajo en menores } & Trabaja & 13 & 12,1 \\
\hline & No trabaja & 94 & 87,9 \\
\hline \multirow{3}{*}{ Posee red de apoyo } & Sí & 68 & 63,6 \\
\hline & No & 39 & 36,4 \\
\hline & Buena & 75 & 70,1 \\
\hline \multirow[t]{2}{*}{ Calidad en relación con padres } & Regular & 30 & 28,0 \\
\hline & Mala & 2 & 1,9 \\
\hline
\end{tabular}

La escala Test de habilidades para la vida tuvo un Alfa de Cronbach de 0,868 lo cual indica una consistencia buena-adecuada del instrumento. Luego de aplicar esta escala se encontraron correlaciones moderadas por encima de 0,5 y altas en cada una de las 10 dimensiones y sus indicadores (subdominios): Manejo de sentimientos y emociones se correlaciona con los indicadores Regulación emocional y Expresión emocional $(p=0,5)$; Manejo de sentimientos y emociones y Manejo de tensiones y estrés se correlacionan con los subdominios: Regulación emocional y Reconocimiento de fuentes de estrés $(p=0,566)$; Manejo de tensiones y estrés, y Comunicación efectiva y asertiva tienen correlación con los subdominios Humildad y Estrategias específicas $(p=0,5) ; y$ los ítems: Respirar profundamente y Tener pensamientos saludables $(p=0,605)$; Toma de decisiones se correlaciona con los subdominios: Decisiones constructivas y Evaluación de consecuencias $(p=0,534)$; y Toma de decisiones, y Solución de problemas y conflictos tienen correlación con los subdominios: Decisiones constructivas, y Flexibilidad y creatividad $(p=0,504)$. La dimensión Solución de problemas y conflictos se correlaciona 
con Relaciones interpersonales $(p=0,432)$, Pensamiento crítico $(p=0,424)$ y Manejo de sentimientos y emociones $(p=0,547)$ (tabla 2$)$.

Tabla 2.

Correlaciones Dominios y subdominios habilidades para la vida

\begin{tabular}{|c|c|c|c|c|}
\hline ÍTEM & \multicolumn{4}{|c|}{ CORRELACIONES } \\
\hline $\begin{array}{l}\text { Manejo de senti- } \\
\text { mientos y emociones }\end{array}$ & Regulación emocional; $p=0,5$ & Expresión emocional; $p=0,5$ & & \\
\hline $\begin{array}{c}\text { Manejo de tensiones } \\
\text { y estrés }\end{array}$ & Regulación emocional; $p=0,5$ & $\begin{array}{c}\text { Reconocimiento de fuentes de } \\
\text { estrés; } p=0,566\end{array}$ & $\begin{array}{l}\text { Decisiones perso- } \\
\text { nales }(p=0,5)\end{array}$ & $\begin{array}{c}\text { Reconocimiento de fuentes } \\
\text { de estrés }(p=0,5)\end{array}$ \\
\hline $\begin{array}{c}\text { Comunicación efecti- } \\
\text { va y asertiva }\end{array}$ & Humildad; $p=0,5$ & Estrategias específicas; $p=0,605$ & & \\
\hline Toma de decisiones & $\begin{array}{l}\text { Decisiones constructivas; } \\
\qquad p=0,534\end{array}$ & $\begin{array}{l}\text { Evaluación de consecuencias; } \\
\qquad p=0,534\end{array}$ & $\begin{array}{l}\text { Decisiones perso- } \\
\text { nales }(p=0,5)\end{array}$ & $\begin{array}{c}\text { Reconocimiento de fuentes } \\
\text { de estrés }(p=0,5)\end{array}$ \\
\hline $\begin{array}{l}\text { Solución de } \\
\text { problemas }\end{array}$ & $\begin{array}{l}\text { Decisiones constructivas; } \\
\qquad p=0,504\end{array}$ & Flexibilidad; $p=0,504$ & & \\
\hline $\begin{array}{l}\text { Solución de } \\
\text { conflictos }\end{array}$ & Creatividad; $p=0,504$ & $\begin{array}{l}\text { Relaciones interpersonales; } \\
\qquad p=0,432\end{array}$ & $\begin{array}{l}\text { Pensamiento críti- } \\
\quad c 0 ; p=0,424\end{array}$ & $\begin{array}{l}\text { Manejo de sentimientos y } \\
\text { emociones; } p=0,547\end{array}$ \\
\hline $\begin{array}{l}\text { Pensamiento } \\
\text { creativo }\end{array}$ & Innovación $(p=0,523)$ & $\begin{array}{l}\text { Iniciativa e Imaginación y Estética } \\
\qquad(p=0,501)\end{array}$ & $\begin{array}{l}\text { Ofrecer ideas para } \\
\text { solucionar proble- } \\
\text { mas }(p=0,580)\end{array}$ & $\begin{array}{l}\text { Esfuerzo por crear proyec- } \\
\text { tos y acciones innovadoras } \\
\qquad(p=0,580)\end{array}$ \\
\hline Pensamiento crítico & $\begin{array}{l}\text { Análisis objetivo de las situa- } \\
\text { ciones }(p=0,523) ; \text { Innovación } \\
(p=0,523) \text {; }\end{array}$ & $\begin{array}{l}\text { Postura crítica ante hechos } \\
\text { sociales }(p=0,537) ; \text { Capacidad } \\
\text { argumentativa }(p=0,537)\end{array}$ & $\begin{array}{l}\text { Manejo de senti- } \\
\text { mientos y emocio- } \\
\text { nes }(p=0,556)\end{array}$ & $\begin{array}{l}\text { Innovación }(p=0,519) \\
\text { Iniciativa }(p=0,519)\end{array}$ \\
\hline
\end{tabular}

Las dimensiones Toma de decisiones y Manejo de tensiones y estrés se correlacionan con los subdominios: Decisiones personales y Reconocimiento de fuentes de estrés $(p=0,5)$; el Pensamiento creativo tuvo correlación con los subdominios: Iniciativa e Imaginación y Estética $(p=0,501)$, e Innovación $(p=0,523)$ y con los ítems: Ofrecer ideas para solucionar problemas y, Esfuerzo por crear proyectos y acciones innovadoras $(p=0,580)$. El Pensamiento crítico y el Pensamiento creativo fueron correlativos a los subdominios: Análisis objetivo de las situaciones, e Innovación ( $p=0,523)$; Pensamiento crítico presentó correlación con Manejo de sentimientos y emociones $(p=0,556)$ y los subdominios: Innovación e Iniciativa $(p=0,519)$ y Postura crítica ante hechos sociales $y$, Capacidad argumentativa $(p=0,537)$ (tabla 2$)$. Las dimensiones Conocimiento de sí mismo y Empatía presentaron correlaciones bajas. Es así que Conocimiento de sí mismo se correlacionó con Manejo de tensiones y estrés ( $p=0,373)$, mientras que Empatía lo hizo con Solución a problemas y conflictos $(p=0,338)$ y Manejo de tensiones y estrés $(p=0,310)$. Se presentaron correlaciones muy bajas entre Habilidades para la vida y Riesgo psicosocial, siendo representativa la correlación entre Pensamiento crítico con Riesgos comunitarios $(p=-0,228)$, y Toma de decisiones con conflictos con Riesgo Relacional $(p=-0,219)$. Las correlaciones entre los tipos de riesgo psicosocial 
fueron muy bajas, de ellas resalta la correlación entre Riesgo familiar y Riesgo Psicológico $(p=-0,228)$ y de esta última con Riesgos Comunitarios $(p=0,275)$ y Riesgos Personales $(p=0,231)$.

Respecto a los riesgos psicosociales los adolescentes identifican riesgos con una cronicidad de "algunas veces" en el riesgo relacional (81,3\%), riesgos intergrupales $(95,3 \%)$, riesgos personales $(97,2 \%)$, riesgo psicológico $(96,3 \%)$, y riesgos comunitarios (96,3\%), mientras que el valor de "nunca" puntuó para la percepción de riesgos ambientales $(97,2 \%$ ) y familiares $87,9 \%$. En relación a las habilidades para la vida se encontró que el conocimiento de sí mismo $(61,7 \%)$, la comunicación efectiva y asertiva $(52,3 \%)$, y las relaciones interpersonales (49,5\%), tienen una cronicidad de "casi nunca", mientras que aquellas habilidades que puntúan "a veces" son empatía $(65,4 \%)$, toma de decisiones (57,9\%), solución de problemas y conflictos $(62,6 \%)$, pensamiento creativo $(53,3 \%)$, pensamiento crítico $(51,4 \%)$, manejo de sentimientos y emociones $(59,8 \%)$ y manejo de tensiones y estrés (46,7\%) (Tabla 3$)$. En $11^{\circ}$ prevalecen "a veces" las siguientes habilidades: Toma decisiones (24,3\%), solución de problemas y conflictos $(23,4 \%)$, pensamiento creativo $(23,4 \%)$, pensamiento crítico $(25,2 \%)$, mientras que en $10^{\circ}$ prevalece el conocimiento de sí mismo (29,9\%), empatía ( $28 \%)$, comunicación efectiva y asertiva $(23,4 \%)$, relaciones interpersonales $(20,6 \%)$, pensamiento creativo $(22,4 \%)$, pensamiento crítico $(24,6 \%)$, manejo de sentimientos y emociones $(25,2 \%)$, y manejo de tensiones y estrés $(17,8 \%)$.

Tabla 3.

Cronicidad de uso de Habilidades para la vida e identificación de riesgos psicosociales

\begin{tabular}{cccc}
\hline \multicolumn{2}{c}{ RIESGOS PSICOSOCIALES } & \multicolumn{2}{c}{ HABILIDADES PARA LA VIDA } \\
\hline TIPO DE RIESGO & ALGUNAS VECES & TIPO DE HABILIDAD & A VECES \\
\hline Riesgo relacional & $81,30 \%$ & Empatía & $65,40 \%$ \\
Riesgo Intergrupal & $95,30 \%$ & Toma de decisiones & $57,90 \%$ \\
Riesgo personal & $97,20 \%$ & Solución de problemas y conflictos & $62,60 \%$ \\
Riesgo Psicológico & $96,30 \%$ & Pensamiento creativo & $53,30 \%$ \\
Riesgo Comunitario & $96,30 \%$ & Pensamiento crítico & $51,40 \%$ \\
Riesgo Ambiental & Nunca $97,2 \%$ & Manejo de sentimientos y emociones & $59,80 \%$ \\
Riesgo Familiar & Nunca 87,9 $\%$ & Manejo de tensiones y estrés & $46,70 \%$ \\
\hline
\end{tabular}

La identificación de riesgos ambientales de acuerdo a los grados cursados es prevalente en el $10^{\circ}$ : ambientales (35,5\%), psicológicos (40,2\%), comunitarias $(38,3 \%)$, personales $(40,2 \%)$, intergrupales $(41,1 \%)$, y familiares $(37,4 \%)$, la relación entre identificación de riesgos y estado civil de los padres revela 
que es mayor y en grado "a veces" cuando estos se encuentran separados: riesgo ambiental $(55,1 \%)$, relacional $(46,7 \%)$, psicológico $(56,1 \%)$, comunitario $(56,1 \%)$, personales $(55,1 \%)$, intergrupales $(54,2 \%)$, y familiares $(47,4 \%)$, la relación entre habilidades para la vida y la calidad de la relación con los padres fue prevalente en la empatía $(44,9 \%)$, solución de problemas y conflictos $(45,8 \%)$, pensamiento crítico $(41,1 \%)$, manejo de sentimiento y emociones (45,8\%). La calidad de la relación buena con los padres genera una mayor identificación del riesgo con una cronicidad de "algunas veces" en los riesgos de tipo: relacional (57,9\%), psicológico (66,4 \%), comunitario (67,3\%), personal (67,3\%), intergrupal (67,3\%), mientras el valor "nunca" es elevado en Riesgo ambiental (67,3\%) y familiar (59,8 \%). Entre la habilidad para la vida "comunicación efectiva y asertiva" y los diversos riesgos se encontró relación con: riesgo personal (51,4\%), ambiental (51,4\%), relacional (43\%), familiar (47,7\%), psicológico (52,3\%), comunitario de "algunas veces" $(41,1 \%)$. Los riesgos de tipo ambiental $(47,7 \%)$, relacional $(42,1 \%)$ psicológico $(48,6 \%)$, comunitario (48,6\%), personal (47,7\%), familiar $(41,1 \%)$ e intergrupal $(45,8 \%)$ tienen un valor de "casi nunca" respecto a la habilidad para la vida "relaciones interpersonales sólidas".

\section{DISCUSIÓN}

Las correlaciones demuestran la existencia de factores protectores en la muestra seleccionada, los cuales se encuentran relacionados a tres elementos: la función ejecutiva toma de decisiones, la respuesta emocional ante eventos estresores y las dificultades en el entorno familiar y académico. Estudios como los de Ochoa (2017) y Ruiz (2012) reiteran estos hallazgos en adolescentes, tomando como base aspectos como la conducta social, toma de decisiones, juicio ético y la moral. Igualmente, la función ejecutiva como capacidad de controlar voluntariamente el pensamiento y la conducta se evidencia como un aspecto clave y protector en la población adolescente. Al respecto es preciso mencionar que dichas funciones tienen componentes de naturaleza cognitiva y emocional que juegan un papel fundamental en la regulación de la conducta encaminada a un objetivo (Lezak, 2004; VerdejoGarcía \& Bechara, 2010; Korzeniowski, 2011; Calle, 2014). El hecho de no ser óptima la correlación entre las dimensiones: manejo de sentimientos, comunicación asertiva y decisiones constructivas implica que hay diferencias entre unos sujetos y otros, hecho que podría indicar que entre más alta la correlación entre estas, menor es el riesgo de tomar decisiones inadecuadas como el suicidio. A ello, debe sumarse el hecho que toda adolescencia es conflictiva per se, puesto que el adolescente busca incluir el estilo de vida 
de los adultos, por lo que en dicha transición se enfrenta a muchos retos y dilemas conflictivos " $y$ entre ellos el más importante es abandonar su niñez para acoplarse al mundo adulto, aspecto que desata crisis porque la mayoría de adolescentes desea obtener beneficios de "ser grande" sin abandonar los beneficios de "ser un niño" (Andrade, 2017, p. 12).

La investigación reveló que en los adolescentes el manejo de sentimientos y emociones se encuentra íntimamente relacionado con la regulación y expresión de las emociones, de modo que quien aprehende afectivamente a reconocer su vida emocional cuenta con mejores habilidades para identificarlas, aplicarlas asertivamente y expresarlas de manera coherente en relación a la toma de decisiones. En este sentido, se encontró que dicha toma de decisiones influye positivamente en la capacidad para solucionar problemas y conflictos, cuando se implementan decisiones constructivas, al tiempo que siendo flexibles y creativos en su ejecución (ver imagen 1). Estudios indican que estas habilidades referencian un adecuado desarrollo de la inteligencia emocional, que permite la implementación de actividades ajustadas a los contextos e interacciones, bajo el reconocimiento de los cambios y estados emocionales propios y ajenos, de acuerdo a situaciones que generan diversos elementos de estrés, presión o tensiones (Extremera \& Fernández, 2003; Berrocal \& Pacheco, 2005; Salguero, Fernández, Ruiz, Castillo, \& Palomera, 2015), lo que quiere decir que "los adolescentes con una mayor habilidad para reconocer los estados emocionales de los demás informaron de mejores relaciones sociales con iguales y padres, menor tensión en sus relaciones sociales, así como de un mayor nivel de confianza y competencia percibida" (Salguero, et al., 2015, p. 143). Lo anterior tiene un efecto importante en el manejo de la vida emocional de los adolescentes, situación visible a través del siguiente esquema (Imagen 1) generado a partir de la información recogida de los adolescentes evaluados.

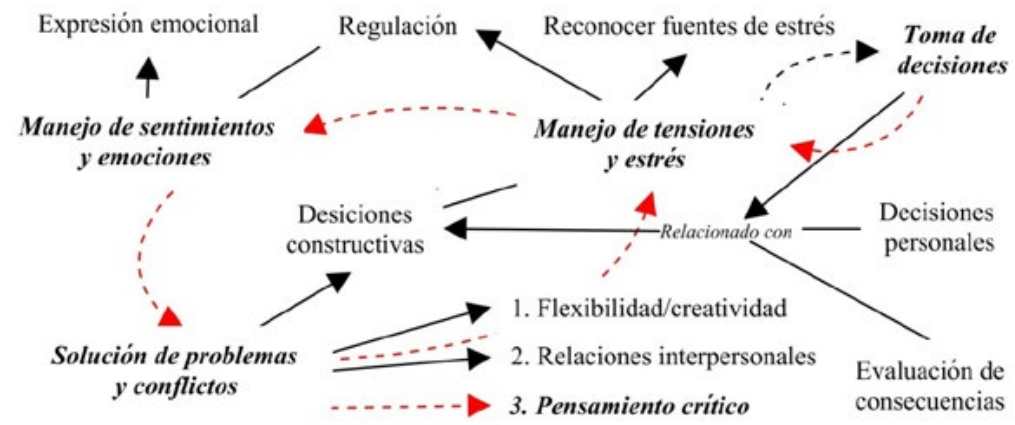

Imagen 1. Relación Manejo sentimientos-emociones-decisiones-conflictos 
Para los adolescentes evaluados el manejo de tensiones y estrés está relacionado con el uso de estrategias específicas como, por ejemplo: respirar profundamente y traer a su mente pensamientos saludables. Respecto al conocimiento de las fuentes de estrés fue visible que estas se encuentran relacionadas con la toma de decisiones, y el manejo que los adolescentes hacen de las tensiones, de allí que, las experiencias de estrés y más que ello, la forma en que son confrontadas representan los principales elementos que determinan la intensidad y las cualidades disfuncionales y funcionales de dichas expresiones emocionales. Para los adolescentes examinar la fuente de estrés alivia las tensiones, y permite la regulación emocional, además del reconocimiento y buen gobierno de la vida emocional. En torno a ello Villarreal, Sánchez y Musitu (2010) indican que la mejoría de las habilidades sociales en los adolescentes implica un buen manejo del estrés, resolución de problemas a través de la objetividad y la comprensión socio-familiar, además de otros elementos que pueden estar en equilibrio relativo tales como, asertividad y autoestima. También indican que contar con un adecuado proyecto de vida, donde se sientan apoyados por sus padres o figuras de autoridad, redunda en conductas positivas de autocontrol y toma adecuada de decisiones. Sánchez y Fernández (2013) agregan a ello el fortalecimiento de las habilidades para la vida y una adecuada resiliencia, la cual se hace fuerte en escenarios familiares, y se robustece en el ámbito educativo.

Así mismo la comunicación efectiva y asertiva guarda relación con la posibilidad de manejar la tensión y el estrés aplicando estrategias específicas de confrontación al tiempo que, conservando una actitud de humildad; en este sentido los adolescentes pueden tomar decisiones constructivas cuando logran evaluar las consecuencias de sus actos. Al respecto, Castro (2005) opina que aquello que los adolescentes entienden por felicidad y toma de decisiones implica una actitud de humildad, es decir, de ser sencillo y aceptar los cambios y responsabilidades que dichas decisiones conllevan. Igualmente, Aguirre (2016) señala que ambos elementos -comunicación efectiva y asertiva- configuran habilidades positivas para la vida, útiles para prevenir factores de riesgo socio-ambientales, al tiempo que sirven de puntos de apoyo para la rehabilitación de adolescentes que han consumido drogas o que se encuentran en situación de vulnerabilidad. A este aspecto deben añadirse los dilemas morales asociados a la capacidad para resolver conflictos y a comunicar sus diversos estados emocionales, mismos que pueden influir en su desarrollo emocional y educativo, y que tienen como referente la implementación de las habilidades para la vida, tanto como la percepción adecuada de los riesgos a los cuales se ven constantemente 
expuestos (Cháidez, \& González, 2013). Los puntajes promedio encontrados en los adolescentes respecto a la relación entre pensamiento creativo y pensamiento crítico, revelan la necesidad de comprender las razones asociadas a su desarrollo, puesto que, mientras el primero cuenta con elementos como Iniciativa e Imaginación y estética, el segundo requiere ser creativo en tanto Análisis de objetivos, Innovación e Iniciativa (Imagen 2).

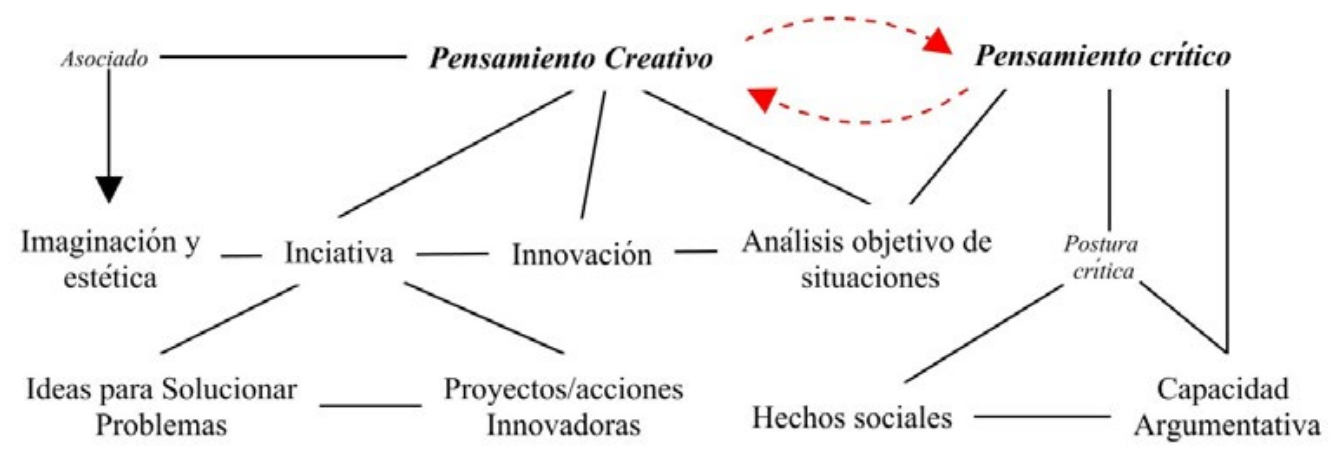

Imagen 2. Relación Pensamiento creativo-pensamiento crítico.

No obstante, el estudio encontró que en aquellos estudiantes que tienen un buen desarrollo de ambos, lo que determina su consolidación es la conservación de una postura crítica ante hechos sociales, un adecuado nivel de auto cuestionamiento y el desarrollo constante de la capacidad argumentativa. Al respecto Ramos (2010) opina que existe una relación importante entre habilidades cognitivas, aprendizaje y pensamiento crítico, y que dichos elementos demandan el desarrollo de un pensamiento cada vez más creativo; de allí que aquellos adolescentes que no desarrollan dichas habilidades, se tornen proclives a experimentar múltiples factores de riesgo psicosocial y ambiental, además de tener constantes problemas con la autoridad familiar. Análogamente, Limiñana (2008) opina que el pensamiento creativo requiere libertad y confianza para tornarse crítico y efectivo en la resolución de problemas. En este sentido, Choque y Chirinos (2009) indican que la toma de decisiones es una habilidad que conlleva un pensamiento crítico capaz de valorar e identificar conflictos, problemas o situaciones que demanden la toma efectiva de decisiones; asimismo revelan que ello requiere de un pensamiento creativo con el que se examine y sintetice lo juzgado, "además de la capacidad de resolver un problema para encontrar alternativas de solución.

La toma de decisiones implica, además, elegir la mejor opción para solucionar una dificultad y tener la facultad para comprender entre varias opciones cuál es la mejor" (p.179). La percepción de los riesgos psicosociales en los 
adolescentes es baja, ello se debe en gran medida a la dificultad de reconocer riesgos intrafamiliares, pues en contraste logran distinguir mejor los riesgos comunitarios, intergrupales y relacionales. Quizá ello sea porque en la familia exista una normalización de las conductas incluso de carácter disfuncional. Al respecto, Satir (1998) argumenta que las familias disfuncionales suelen regularizar conductas anómalas haciendo que sean parte de su normalidad. Asimismo, Bowen (1991) señala que ello se debe a la diferenciación que existe en el sí mismo de cada integrante del sistema familiar; así, para algunos estas conductas serán normofuncionales y pueden llegar a legitimarlas como válidas, mientras que para otros puede constituir un elemento permanente de conflicto. Respecto a las habilidades para la vida, la investigación encontró como datos relevantes, que aquellas $\mathrm{HV}$ relacionadas con el autoconocimiento, solución de problemas y conflictos, empatía, comunicación efectiva y asertiva, manejo de sentimientos y emociones, y pensamiento crítico y creativo, suelen ser las de mayor desarrollo, lo cual llama la atención dado que, en su mayoría pueden referenciar habilidades de mayor desarrollo en el ámbito exo-familiar.

Al respecto, Rice (2000) considera que el desarrollo social es necesario en la adolescencia, pero que debe ser análogo al desarrollo positivo a nivel intrafamiliar; de allí que cuando haya un desequilibrio o falta de interrelación entre ambos desarrollos, se produzca un desajuste que puede conllevar a crisis internas. Igualmente, Sánchez, Bilbao, Rebollo y Barón (2001), señalan que el desarrollo afectivo y social del adolescente depende en gran medida de las interacciones familiares y sociales que este tiene en su familia cercana, extensa y con su entorno. Para Rice (1997), el desarrollo humano se produce de manera escalar, y de acuerdo a ciclos vitales específicos en los cuales prima la cualidad y claridad de las interacciones entre los sujetos; de allí que un adecuado desarrollo en el adolescente implique relaciones humanas gratificantes de apoyo, de respeto y solidaridad entre sus miembros además de niveles de acogimiento y sostén positivo ante situaciones adversas. Para el adolescente es trascendental el soporte de sus padres y de su familia al igual que de su familia extensa (Berk, 1999) con el fin de desarrollar sus habilidades vitales (Mangrulkar et al., 2001), además de otras habilidades para la identificación y afrontamiento de riesgos (Urzúa \& Correa, 1998).

\section{CONCLUSIONES Y RECOMENDACIONES}

El pensamiento creativo y crítico presenta un puntaje alto entre $10^{\circ}$ y $11^{\circ}$ y es coadyuvante en la constitución de habilidades importantes a desarrollar en dichos períodos; el hecho de que se presente más bajo en el grado previo, 
se puede deber a la maduración cortical propia de la etapa escolar media luego de los 15 años. Los tres grados de escolaridad tienen puntajes altos en el reconocimiento de los riesgos "comunitarios, personales, intergrupales"; este parece estar asociado a los conocimientos empíricos y vivencias de su entorno, el cual presenta estos riesgos como parte de su configuración socio-cultural. El grupo étnico, al igual que la religión, el trabajo y el estrato no se constituyeron en variables determinantes para la identificación de riesgos y el fortalecimiento de las habilidades para la vida. Se encontró que "no-trabajar" es un elemento que fortalece las habilidades para la vida. El trabajo en menores no fue un factor significativo para determinar que las habilidades para la vida sean fuertes o no lo sean; tampoco se evidencia como un factor de riesgo asociado a los riesgos mencionados. Otro hallazgo es que entre mejor es la relación con sus padres, mejores son sus habilidades para la vida y la implementación que se hace de ella en los diferentes ámbitos sociales.

Un hecho interesante es que los hijos de padres separados generan mayores habilidades para reconocer riesgos, esto puede ser porque la mayoría de ellos debe asumir roles y responsabilidades a muy temprana edad, y porque suelen aprender de esta experiencia, modos particulares de resolver conflictos y de tratar con la frustración, factor que se encuentra determinado por las habilidades para la vida que desarrollen durante dicho periodo. Cuando la calidad en la relación con padres es buena, el reconocimiento del riesgo familiar y ambiental es bajo, mientras que la identificación de riesgos de tipo relacional, intergrupal, personal, psicológico y comunitario es mayor, lo cual no los torna exentos de problemas de relación, precisamente por el grado de cercanía y conflicto que la relación padres-hijos implica. De suyo, dicha relación adecuada permite identificar con mayor facilidad los riesgos. Es importante mencionar que dentro de una vinculación sana debe existir algo de conflicto y que resolverlos conjuntamente forma parte de las habilidades vitales de todo ser humano. El reconocimiento de los riesgos se afecta en la interacción con los padres, lo cual disminuye sus habilidades para la vida, mismas que le sirven para manejar las relaciones intrafamiliares como para sentir una confianza elevada en su entorno social y familiar; este resultado es el indicador de una ambivalencia -no siempre visible- en las interacciones, pues a menudo los mayores riesgos para la salud mental y física de los adolescentes provienen del hogar.

No obstante, dicha paradoja no de forma necesaria debe ser negativa, ya que puede estar asociada a la capacidad de las familias para sortear lo adverso 
y aprender de las tensiones y conflictos, lo que a su vez es indicador del desarrollo y fortalecimiento de habilidades para la vida al interior del hogar. La familia resulta crucial para determinar el tipo de habilidades adquiridas y se constituye en la fuente de aprendizaje, robustez y entrenamiento en dichas habilidades a lo largo del ciclo vital. Se halló que la identificación de riesgos psicosociales es mayor en $10^{\circ}$ (familiar, ambiental, intergrupal, personal, psicológico y comunitario) mientras que el desarrollo de las habilidades para la vida es mayor en $11^{\circ}$, especialmente aquellas relacionadas con la toma de decisiones, solución de problemas y conflictos, pensamiento creativo y crítico. Se encontró que la identificación de los riesgos intergrupales y familiares, disminuye cuando se fortalece el conocimiento de sí mismo, y que la capacidad de reconocer el riesgo ambiental, relacional, psicológico, intergrupal y familiar bajan si se fortalece la comunicación efectiva/asertiva; de allí que en las relaciones interpersonales fuertes disminuya la probabilidad de reconocer cualquier riesgo debido al grado de confianza que la relación sugiere.

Igualmente cabe concluir que la toma de decisiones no fue un elemento significativamente influyente en la identificación de los riesgos evaluados. No obstante, dicha función ejecutiva se vincula con otros procesos del desarrollo a nivel social, por tanto, habría que revisar la interacción de estas con las otras variables como la calidad de los vínculos parentales. Estos hallazgos estadísticos alrededor de las dimensiones pensamiento crítico, solución de problemas y conflictos, indican que es muy importante la autorregulación, la introspección y en general el funcionamiento ejecutivo como factores protectores de los adolescentes ante la presencia de conflictos sociales, familiares e internos. Es decir, que en la medida que los menores recurren a estas dimensiones como estrategias en el momento de tomar decisiones, las posibilidades de actuar de manera sana en términos de salud mental aumentarán y las posibilidades de respuesta patológica como la suicida disminuirán.

Financiamiento: Este artículo es resultado de la investigación: "Relación entre habilidades para la vida y factores protectores del suicidio en adolescentes escolarizados" realizada como proyecto de convocatoria M3828 en la Universidad de San Buenaventura, Medellín.

Agradecimientos: A la Universidad de San Buenaventura por su apoyo en los proyectos de investigación y a las instituciones educativas que facilitaron el acceso a la población y la aplicación de instrumentos. 


\section{REFERENCIAS}

Aguirre, R. A. (2016). Construcción de un proyecto de vida en adolescentes que consumen drogas. Un estudio de casos (Bachelor's thesis). Universidad del Azuay, Cuenca Ecuador. http://dspace.uazuay.edu.ec/handle/datos/5549

Alfonso, B., Calcines, M., Monteagudo de la Guardia, R., \& Nieves, Z. (2015). Estrés académico. Edumecentro, 7(2), 163-178. Recuperado de http://scielo.sld.cu/ scielo.php?pid=S2077-28742015000200013\&script=sci_abstract

Andrade, J. A (2012). Aspectos psicosociales del comportamiento suicida en adolescentes. Revista Electrónica de Psicología Iztacala, 15(2), 688. Recuperado de http://www.medigraphic.com/pdfs/epsicologia/epi-2012/epi122q.pdf

Andrade, J. A. (2017). El adolescente no es el problema. Guía para padres con hijos preadolescentes y adolescentes. Berlín: Editorial académica española.

Andrade, J. A., \& Gonzales, J. A. (2012). Asociación entre el consumo de sustancias psicoactivas y actividad delictiva en adolescentes. Poiesis, 1(1), 1-10. https://doi. org/10.21501/16920945.314

Bahamón, M. J., Alarcón-Vásquez, Y., Reyes, L., Trejos, A. M., Uribe Alvarado, J. I., \& Galindo, C. (2018). Prácticas parentales como predictoras de la ideación suicida en adolescentes colombianos. Psicogente, 21(39), 50-61. https://dx.doi. org/10.17081/psico.21.39.2821

Berk, L. E. (1999). Desarrollo del niño y el adolescente. Barcelona: Prentice Hall Iberia.

Berrocal, P. F., \& Pacheco, N. E. (2005). La Inteligencia Emocional y la educación de las emociones desde el Modelo de Mayer y Salovey. Revista Interuniversitaria de Formación del profesorado, 1(54), 63-94. Recuperado de https://www.redalyc. org/articulo.oa?id=27411927005

Bowen, M. (1991). De la familia al individuo: la diferenciación del sí mismo en el sistema familiar. Primera edición. México: Ediciones PAIDÓS.

Bravo, H. A. (2005). La iniciativa de habilidades para la vida en el ámbito escolar. Revista del Instituto de Investigaciones Educativas, 9(16), 25-34. Recuperado de http://sisbib.unmsm.edu.pe/bibvirtualdata/publicaciones/inv_educativa/2005_ n16/a002.pdf

Bravo, H. A., \& Garzón, G. A. (2010). Habilidades para la vida. En búsqueda del desarrollo del ser humano integral. En J. Ippolito-Shepherd, Promoción de la salud. Experiencias internacionales en escuelas y universidades (pp. 123-139). Buenos Aires: Paidós.

Calle, D. (2014). Cerebro y cognición social: un puente entre la neurociencia y la construcción social del sujeto. Realitas, 2(1), 51-56. 2014. Recuperado de https:// www.researchgate.net/publication/326353700_Cerebro_y_cognicion_social_ Un_puente_entre_la_neurociencia_y_la_construccion_social_del_sujeto_ Brain_and_social_cognition_A_bridge_between_neuroscience_and_the_social_ construction_of_the_subject

Calle, D. (2016). Génesis neuropsicológica de las funciones ejecutivas. Capítulo 3 en De las neurociencias a la neuropsicología. J. Ávila-Toscano \& D. Da Silva. De las neurociencias a la neuropsicología: el estudio del cerebro humano. Barranquilla: Editorial Unirefromada

Calle, D., \& Gonzáles, J. (2016). Funciones ejecutivas, empatía y conducta suicida: Lo que nos cuenta la neurociencia de la cognición social. En J. Carmona, J. Andrade \& D. Calle (Eds.), Ensayos académicos en torno al suicidio (pp. 37-50). Armenia: Editorial Fundación Participar IPS. 
Castro, A., \& Casullo, M. (2000). Rasgos de personalidad, bienestar psicológico y rendimiento académico en adolescentes argentinos. Interdisciplinaria, 18(1), 65-85. https://www.redalyc.org/pdf/180/18011326003.pdf.

Castro, J. L. E. (2005). Análisis de problemas y toma de decisiones. México D.F.: Pearson Educación.

Casullo, M., Bonaldi, P., \& Fernández, M. (2000). Comportamientos suicidas en la adolescencia: morir antes de la muerte. Buenos Aires: Lugar Editorial.

Cháidez, M. L. \& González, C. S. (2013). Los dilemas morales en la lectoescritura del cuento en jóvenes de educación media superior. XII Congreso Nacional de Investigación Educativa COMI Memorias.

Choque, R., \& Chirinos, J. L. (2009). Eficacia del Programa de habilidades para la vida en adolescentes escolares de Huancavelica, Perú. Revista de Salud pública, 11(2), https://doi.org/10.1590/S0124-00642009000200002

Díaz, L. E., Rosero, R. F., Melo, M. P. \& Aponte, D. (2013). Habilidades para la vida: análisis de las propiedades psicométricas de un test creado para su medición. Revista Colombiana de Ciencias Sociales, 4(2), 181-200. Recuperado de https:// dialnet.unirioja.es/servlet/articulo?codigo $=5123783$

Espada, J., Méndez, X., Griffin, K., \& Botvin, G. (2003). Adolescencia: consumo de alcohol y otras drogas. Papeles del Psicólogo, 23(84), 9-17. https://www.researchgate.net/publication/237040144_Adolescencia_Consumo_de_alcohol_y_ otras_drogas

Extremera, N., \& Fernández-Berrocal, P. (2003). La inteligencia emocional en el contexto educativo: hallazgos científicos de sus efectos en el aula. Revista de educación, 332(2003), 97-116. Recuperado de https://dialnet.unirioja.es/servlet/ articulo? codigo $=776719$

Farberow, N. L. (1980). Las muchas caras de suicidio. Nueva York: Prentice-Hall

Ferguson, D. M., Beautrais, A. L. \&. Horwood, L. J. (2003). Vulnerability and resiliency to suicidal behaviours in young people. Psychological Medicine, 33(1), 61-73. Recovered https://www.ncbi.nlm.nih.gov/pubmed/12537037

Goldney, R.D., Winefield, A.H., Tiggemann, M., Winefield, H.R. \& Smith, S. (1989). Suicidal ideation in a young adult population. Acta Psychiatrica Scandinavica, 79(5), 481-9. Recovered https://www.ncbi.nlm.nih.gov/pubmed/2750549

Góngora, V. \& Casullo, M. (2009). Factores protectores de la salud mental: un estudio comparativo sobre valores, autoestima e inteligencia emocional en población clínica y población general. Interdisciplinaria, 26(2), 183-205. Recuperado de http:// www.scielo.org.ar/scielo.php?script=sci_arttext\&pid=S1668-70272009000200002

Gonzales, J., Gil, J., Hernández, D. \& Henao, L. (2016). Evaluación de las expectativas negativas y tipo de riesgo suicida en estudiantes de $9^{\circ}, 10^{\circ}$ y $11^{\circ}$ de una institución educativa del departamento del Quindío. Duazary, 13(1), 7-14. Recuperado de http://revistas.unimagdalena.edu.co/index.php/duazary/article/view/1582

Henriksson, M. H., Henriksson, M.M., Aro, H.M., Mattumen, M.J. Heikkimen, M.E. Isontsa, E.T., Kuoppasalmi, K. \& Löngvst, J.K. (1993). Mental disorders and comorbidity in suicide. American Journal Pychychiatry, 150, 935-40. Recovered https:// www.ncbi.nlm.nih.gov/pubmed/8494072

Herrera, P. M., \& Avilés, K. (2000). Factores familiares de riesgo en el intento suicida. Revista Cubanade Medicina GeneralIntegral,16(2),134-137. Recuperadode http:// scielo.sld.cu/scielo.php?script=sci_arttext\&pid=\$0864-21252000000200005

Isometsä, H. A. (2001). Psychological autopsy studies--a review. Eur Psychiatry, 16(7), 379-385. Recovered https://www.ncbi.nlm.nih.gov/pubmed/11728849 
Korzeniowski, C. G. (2011). Desarrollo evolutivo del funcionamiento ejecutivo y su relación con el aprendizaje escolar. Revista de Psicología, 7(13), 7-26. Recuperado de http://bibliotecadigital.uca.edu.ar/repositorio/revistas/desarrollo-evolutivo-funcionamiento-ejecutivo

Lezak, M.D. (2004). Neuropsychological assessment. New York: Oxford University Press.

Limiñana, R. M. (2008). Cuando crear es algo más que un juego: Creatividad, Fantasía e Imaginación en los jóvenes. Cuadernos de la Facultad de Humanidades y Ciencias Sociales. Universidad Nacional de Jujuy, 1(35), 39-43. Recuperado de http://www. scielo.org.ar/scielo.php?script=sci_abstract\&pid=\$1668-81042008000200003

Lonnquist J. (2002). Proyecto de prevención del suicidio. Rev Psychiatria, 12(23), 35-51. Recuperado de http://apps.who.int/iris/bitstream/10665/107452/1/ E77922.pdf

Mangrulkar, L., Whitman, C. V., \& Posner, M. (2001). Enfoque de habilidades para la vida para un desarrollo saludable de niños y adolescentes. Organización Panamericana de la Salud -OPS. Informe Anual

Mantilla, L. (2001). Habilidades para la vida. Una propuesta educativa para la promoción del desarrollo humano y la prevención de problemas psicosociales. Tercera edición. Bogotá: Fe y Alegría.

Mantilla, L. \& Chahín, I. (2006). Habilidades para la vida. Manual para aprenderlas y enseñarlas. Bilbao: EDEX.

Mardomingo, M. J., \& Catolin, M. L. (1992). Intentos de suicidio en la infancia y adolescencia: características epidemiológicas. An ESP Pediatría, 37(1), 29-32. http:// scielo.sld.cu/scielo.php?script=sci_arttext\&pid=S0864-21251998000600008

Maris, R. W. (1992). Overview of the Study of Suicide Assessment and Prediction. In R.W. Maris, A.L. Berman, J.T. Maltsberg, \& R.I. Yufit (Editores), Assessment and Prediction of Suicide. (pp.3-23) New York: The Guilford Press. https://doi. org/10.1002/depr.3050010510

Ochoa, C. (2017). Estudio sobre la toma de decisiones, el funcionamiento ejecutivo, la impulsividad en el juego patológico. (Tesis doctoral). Universidad de Barcelona. Recuperado de http://diposit.ub.edu/dspace/bitstream/2445

Omar, A., Almeida da Silva Junior, S. H., Paris, L., Aguiar de Souza, M., \& del Pino Peña, R. (2010). Resiliência e enfrentamento do estresse em adolescentes: efeitos mediadores dos valores culturais. Psicologia em Revista, 16(3), 448-468. http://pepsic. bvsalud.org/scielo.php?script=sci_arttext\&pid=S1677-11682010000300003

Quiceno, J. M., \& Vinaccia, S. (2013). Calidad de vida, factores salutogénicos e ideación suicida en adolescentes. Terapia Psicológica, 31(2), 263-271 https:// psycnet.apa.org/record/2013-21104-012

Quiceno, J. M., \& Vinaccia, S. (2014). Calidad de vida en adolescentes: Análisis desde las fortalezas personales y las emociones negativas. Terapia psicológica, 32(3), 185-200. Recuperado de https://scielo.conicyt.cl/scielo.php?script=sci_arttex$\mathrm{t} \& \mathrm{pid}=\mathrm{S} 0718-48082014000300002$

Ramos, A. I. (2010). Desarrollo de habilidades cognitivas con aprendizaje móvil: un estudio de casos/Developing Cognitive Skills with Mobile Learning: a Case Study. Comunicar, 17(34), 201-209. http://eprints.rclis.org/17752/

Rice, F. P. (1997). Desarrollo humano: estudio del ciclo vital. Madrid: Pearson Educación.

Rice, F. P. (2000). Adolescencia: desarrollo, relaciones y cultura. Madrid: Prentice Hall. 
Ruiz, I. P. (2012). Estudio de la función ejecutiva en menores infractores de 14 a 16 años del Centro de Adolescentes Infractores (CAl) Virgilio Guerrero. (Tesis de maestría). Universidad Central del Ecuador.

Salguero, J. M., Fernández-Berrocal, P., Ruiz-Aranda, D., Castillo, R., \& Palomera, R. (2015). Inteligencia emocional y ajuste psicosocial en la adolescencia: El papel de la percepción emocional. European Journal of Education and Psychology, 4(2), 143-152. http://www.infocop.es/view_article.asp?id=3938

Sánchez, F. L., Bilbao, I. E., Rebollo, M. J., \& Barón, M. J. (2001). Desarrollo afectivo y social. Barcelona: Ediciones Pirámide.

Sánchez, I. L., \& Fernández, E. H. (2013). El diálogo Habilidades para la Vida-Resiliencia: una hojeada desde el acontecer docente educativo. EduSol, 13(42), 1-12. http://edusol.cug.co.cu/index.php/EduSol/article/view/75

Satir, V. (1998). Relaciones humanas en el núcleo familiar. Sexta edición. México: Editorial PAX.

Urzúa, R. F., \& Correa, M. V. (1998). El adolescente y sus conductas de riesgo. Santiago: Ediciones Universidad Católica de Chile.

Verdejo, A., \& Bechara, A. (2010). Neuropsychology of Executive Functions. Psicothema, 22(2), 227-235. http://www.psicothema.com/psicothema.asp?id=3720

Villarreal, M. E., Sánchez, J. C., \& Musitu Ochoa, G. (2010). Cómo mejorar tus habilidades sociales. Programa para adolescentes: manejo del estrés, resolución de problemas, autoestima, asertividad, proyecto de vida y administración del tiempo libre. Mexico: Editorial Universidad Autónoma de Nuevo León. https://www. academia.edu/24324206/Libro_habilid_soc

Welch, S. (2001). A review of the literature on the epidemiology of parasuicide in the general population. Psychiatr Serv, 52(3), 368-375. https://doi.org/10.1176/appi. ps.52.3.368

Esta obra está bajo: Creative commons attribution 4.0 international license. El beneficiario de la licencia tiene el derecho de copiar distribuir, exhibir y representar la obra y hacer obras derivadas siempre y cuando reconozca y cite la obra de la forma especificada por el autor o el licenciante.

\section{(cc) $\mathbf{B Y}$}




\section{ANEXOS.}

\section{Ficha de Caracterización}

$\begin{array}{cccc}\text { Edad: } & \text { Género: Masculino () Femenino () } & \text { País de nacimiento: } & \\ \text { Transexual () } & \text { Ciudad de Residencia: } & \\ \text { Grado cursado: } & 9^{\circ}() 10^{\circ}() 11^{\circ}() & \text { Ciudad en la que vive: } & \\ \text { Religión: } & \text { Católica () } & \text { Cristiana () } & \text { Evangélica () Otra: } \\ \text { Sus padres están: } & \text { Separados () } & \text { Viven Juntos () } & \text { No convive con ellos () }\end{array}$

El estrato en el que vive es: $\quad 1() 2() 3() 4() \quad$ La casa en que vive: Propia () Alquilada ()

Vive con: Ambos padres ( ); Uno de los padres ( ); Tíos u otros Adultos ( ); Vive solo ()

Convive con otros familiares en su casa: Abuelos ( ) Tíos ( ) Primos ( ) Otros

Cuántos hermanos tiene? Ninguno () 1 () 2 () 3 () 4 ()

Qué lugar ocupa entre sus hermanos Primero ( ); El del medio ( ); Último ( ); Otro Cuál?

Grupo étnico con el que se identifica: Indígena ( ) Mestizo ( ) Afrocolombiano ( ) Raizal ( ) ROM ( ) Otro

Cuál?

Actualmente tiene un trabajo Sí ( ) No ( ) Cuál?

Cuenta con una red de apoyo/amigos cuando tiene problemas? Sí ( ) No ( )

La relación con sus padres es: Buena ( ); Regular ( ); Mala ( ); A veces Buena y mala () 


\section{Cuestionario de Identificación de riesgos vitales}

\begin{tabular}{|c|c|c|c|c|c|}
\hline & ITEM & NUNCA & $\begin{array}{l}\text { ALGUNAS } \\
\text { VECES }\end{array}$ & $\begin{array}{l}\text { FRECUENTE- } \\
\text { MENTE }\end{array}$ & SIEMPRE \\
\hline 1 & Me agrada fumar cigarrillos & & & & \\
\hline 2 & Consumo bebidas alcohólicas & & & & \\
\hline 3 & Fumo marihuana & & & & \\
\hline 4 & Inhalo una o varias sustancias psicoactivas & & & & \\
\hline 5 & Participo de peleas & & & & \\
\hline 6 & Cuando alguien me ofende o agrede le hago lo mismo & & & & \\
\hline 7 & Le hago Bullying a otros compañeros & & & & \\
\hline 8 & Me hacen Bullying otros compañeros & & & & \\
\hline 9 & $\begin{array}{c}\text { Tengo sentimientos de frustración, infelicidad y } \\
\text { soledad }\end{array}$ & & & & \\
\hline 10 & $\begin{array}{c}\text { Tengo problemas personales que me afectan emocio- } \\
\text { nalmente (depresión) }\end{array}$ & & & & \\
\hline 11 & Tengo poca esperanza en el futuro & & & & \\
\hline 12 & $\begin{array}{c}\text { Siento que pierdo el control a causa de la rabia y la ira } \\
\text { que me causan ciertos problemas }\end{array}$ & & & & \\
\hline 13 & Me alejo de la gente y me agrada estar solo(a) & & & & \\
\hline 14 & $\begin{array}{l}\text { Pienso que en mi barrio cada cual vive su vida sin } \\
\text { importarle los demás }\end{array}$ & & & & \\
\hline 15 & $\begin{array}{l}\text { Mi casa queda en un lugar en el que hay peligros (ven- } \\
\text { ta de droga, bandalismos, robos frecuentes) }\end{array}$ & & & & \\
\hline 16 & Me siento discriminado en mi barrio y/o comunidad & & & & \\
\hline 17 & $\begin{array}{l}\text { Invierto mi tiempo libre en cosas productivas (deporte, } \\
\text { estudiar, ayudar en la casa, arreglar mi cuarto, etc.) }\end{array}$ & & & & \\
\hline 18 & $\begin{array}{l}\text { Suelo hacerme cortes en brazos y piernas (o en otros } \\
\text { lugares) cuando tengo problemas }\end{array}$ & & & & \\
\hline 19 & Uso métodos poco saludables para adelgazar & & & & \\
\hline 20 & Tengo relaciones sexuales de forma irresponsable & & & & \\
\hline 21 & $\begin{array}{c}\text { Soy poco cuidadoso con la información personal que } \\
\text { pongo en las redes sociales }\end{array}$ & & & & \\
\hline 22 & $\begin{array}{c}\text { Me relaciono mejor con amigos y compañeros que } \\
\text { otros tildan de "malos" }\end{array}$ & & & & \\
\hline 23 & Tomo riesgos sin pensar en las consecuencias & & & & \\
\hline 24 & Rompo las reglas y no acato normas & & & & \\
\hline 25 & Mis padres me castigan injustificadamente y sin razón & & & & \\
\hline 26 & $\begin{array}{l}\text { En mi familia se violan las reglas y cada cual hace lo } \\
\text { que quiere }\end{array}$ & & & & \\
\hline 27 & En mi familia existe persiste un trato fuerte o violento & & & & \\
\hline 28 & $\begin{array}{c}\text { Algunos adultos que conozco tienen comportamientos } \\
\text { inapropiados (consumen alcohol, drogas, agresiones, } \\
\text { violencia) }\end{array}$ & & & & \\
\hline
\end{tabular}

